\title{
State-of-the-Art of Commercial Proctoring Systems and Their Use in Academic Online Exams
}

\author{
Simone Arnò, Department of Medicine and Aging Sciences, University of Chieti-Pescara, Chieti, Italy \\ Alessandra Galassi, Department of Life, Health, and Environmental Sciences, University of L'Aquila, L'Aquila, Italy \\ Marco Tommasi, Department of Medicine and Aging Sciences, University of Chieti-Pescara, Chieti, Italy \\ Aristide Saggino, Department of Medicine and Aging Sciences, University of Chieti-Pescara, Chieti, Italy \\ Pierpaolo Vittorini, University of L'Aquila, L'Aquila, Italy
}

\begin{abstract}
Online proctoring generally refers to the practice of proctors monitoring an exam over the internet, usually through a webcam. This technology has gained relevance during the current COVID-19 pandemic, given that the social distance owing to health reasons has consequently led to the switching of all learning and assessment activities to online platforms. This paper summarises the available state-of-the-art of commercial proctoring systems by identifying the main features, describing them, and analysing the way in which different proctoring programs are grouped on the basis of the services they offer. Furthermore, the paper reports on two case studies concerning online exams taken with both automated and human proctoring approaches. The outcomes from state-of-the-art approaches and the experience gained by the two case studies are then summarised in the conclusion, where the need for an organisational effort in loading photographs that can be used to easily recognise student faces, and using an automated online proctoring program to support manual proctoring have been suggested.
\end{abstract}

\section{KEYWORDS}

Automated Proctoring, Comparing Proctoring, Distance Assessment, Human Proctoring, Live Proctoring, Multidimensional Scaling, Online Exam, Proctoring Functions

\section{INTRODUCTION}

In last few years, online courses and exams have become a more common practise, giving students the opportunity to attend the courses and take exams from places outside the physical classroom (O'Reilly \& Creagh, 2015). Educational programs have evolved to provide solutions for students with changing needs, and the widespread adoption of online learning courses by private or public institutions is a further incentive for the development of programs for learning platforms with a reduction of costs for courses and training. Colleges and universities, while adopting these new educational technologies, require a cloud technology called "online proctoring" (O'Reilly \& Creagh, 2016). Online proctoring programs (OLPs), sometimes called remote proctoring, generally refer to the digital techniques for monitoring and controlling student activities during an exam through webcams and internet connections (Hylton, 2016), thus preventing and detecting any possibility of malpractice. OLPs record data through an online service for storing and reviewing student behaviours 
during an exam. Moreover, OLPs usually include functions to authenticate the examinee's identity to verify that this is the actual person taking the examination (O'Reilly \& Creagh, 2015; O'Reilly \& Creagh, 2016). OLPs can be broadly classified into the following non-mutually exclusive categories:

1. Live Proctoring Programs require a person (the proctor) to be in a remote location to control the examinee's activities like a monitor in real time, ensuring the test-taker's authentication and preventing any form of unfair actions. If the examinee indulges in malpractice, the proctor can interrupt the exam. Introduced and tested in 2006, live proctoring started expanding on a largescale in 2008, with a rapid growth of assessments from a few hundred assessed students per month in 2013 to several thousand in 2015 (Shingal, 2020; Foster \& Layman, 2013; O'Reilly \& Creagh, 2015).

2. Recorded Proctoring Programs do not make use of a human proctor to control examinee behaviours during the entire exam. The student behaviours are recorded during the examination. Teachers, professors, or people with proctoring functions must review the recorded video and check the presence of possible flags that signal doubt in an examinee's activities.

3. Automated Proctoring Programs are currently the most advanced programs available. Examinee behaviours are recorded during the test, and an automated system then reviews the feed through advanced audio-video analysis functions to detect any anomalous or illicit activities (Shingal, 2020).

O'Really and Creagh (2016) also reported a similar categorisation of proctoring programs based more on variations in an interactive approach between the program and user. The authors defined (a) a traditional proctoring approach completely based on human invigilators to detect misconduct; (b) a technology-facilitated proctoring approach in which humans are aided by technological support (e.g. AI support); and (c) an automatic proctoring approach based on the use of pattern recognition functions to detect anomalies through automated procedures.

Nevertheless, this technology has gained a higher relevance because of the COVID-19 pandemic owing to which educational activities in schools and universities have been interrupted during the lockdown. The most problematic issue is guaranteeing that proctoring systems have the same quality level as the educational courses and assessment methods provided before the pandemic, when students could physically attend their courses and exams.

There have been relatively few studies on the criteria for choosing a proctoring system, and a few available papers highlight different characteristics or functions of OLPs as the most relevant. Some authors have proposed using webcams as the best device to prevent students from misconduct (Hylton, 2016). Other authors have claimed that OLPs must be chosen on the basis of benefits such as a reliable assessment of examinee competence and saving time, effort, and cost (Okada, 2015). Others have highlighted the influence of OLPs on the educational experience of the students, both positive and negative (Milone, 2017; Weiner, 2017). Finally, a few authors have drawn attention to student performance, stating that OLPs can reduce the quality of the students' performance (Alessio, 2017; Davis, 2016), significantly reduce the time required to finish the exam (Alessio, 2017), and decrease anxiety during the examination (Kolski, 2018).

Because this is a new area of technology, choosing the best OLP can be a time-consuming and difficult task owing to a great variety of systems with different available functionalities. The first contribution of this paper is the identification and summarisation of the features of the main commercial OLP systems currently in use, to compare their various services or functions, and to highlight those characteristics, either present or absent, that characterise the specific OLP system. Based on the available features, we also analysed the similarity or dissimilarity of the identified OLP systems. In addition, we report on two experiences. In the first experience, a few authors used a commercial automated OLP to provide assistance during online exams in two university courses 
of a psychological faculty. In the second experiment, the authors used live proctoring through a videoconferencing platform.

It is worth remarking that the authors could not find literature containing a thorough description of OLP systems and/or the experiences of their application in a real context. The only study providing some information regarding the functions of different OLPs was conducted by Foster and Layman (Foster \& Layman, 2013), who described a limited number of OLP systems. Dating back to 2013, to the best of our knowledge, no other studies focusing on the same argument have been published. By comparing and classifying the OLP systems as well as reporting on two actual case studies, our research can potentially provide private and public educational institutions to help in finding the most suitable solution to a student assessment through online exams.

\section{STATE-OF-THE-ART OF COMMERCIAL PROCTORING SYSTEMS}

\section{Comparison Table}

We searched OLP companies on the Internet to collect all necessary information regarding their functions and offered services. In this research, we followed a method on reporting the OLP characteristics. Initially, we started our research by analysing the general features of the OLP programs. We then moved to the analysis of more specific features. The general features of the OLP programs were obtained using Google and Google Scholar, inserting the keywords "proctoring exams," "online exams," and "proctoring system." All links obtained in this research were then used to collect information regarding the product or service descriptions by analysing white papers and/or video clips describing and illustrating the features of the system. For each identified function of the OLP program, we proceeded with more specific research to highlight the relevant features. Often, the descriptions about a function reported in the developer's websites were lacking or insufficient, and thus we consulted the user manuals or other public resources available on university web portals that have already used the analysed system. Unfortunately, during this part of the research, we found that many proctoring systems do not allow the possibility to download the program for a trial period or do not allow for a simulation on the web. The intention of our research was to gather all publicised features, which are the principal information that buyers use in their purchases, and to give a provide a synopsis of the current features of all analysed OLP systems, to easily compare the services offered by different venders or providers. This task was not easy because of the heterogeneity in the different terminology adopted by the different websites; nevertheless, we defined a global scheme with information on the features of the different OLP systems. These results are reported in Tables 1-3. The names of the identified features/sub-features of each OLP system are listed in the rows, and the names of the OLP systems are listed in the columns. A " $v$ " in a cell indicates that the specific feature is included in the system. When in doubt, the cell was left blank.

The identified features are described below:

- LMS integration: This indicates that the online proctoring system allows easy and quick integration into the existing Learning Management System (LMS), for example, Moodle and Canvas;

- Scalability: That is the ability of a system to increase its performance if new resources are provided to the system;

- Android/iOS secondary device support: The system allows the use of other technologies to detect the student's behaviour and scan the physical room where the student is taking the exam;

- Authentication functions: Identification of the student can take place in different ways, e.g. ID verification (username and password), e-mail, ID documents, facial recognition, voice recognition, fingerprint, recognition of the iris, other biometric measurements, and keystroke analysis; 
- Lockdown functions: The system provides functionality as a lockdown browser, disable copy and paste, disable right click of the mouse, disable new tabs, disable printing, disable screenshot, prevent website access, record web traffic, and lockdown external applications (e.g., Skype);

- Monitoring functions: the system guarantees the possibility to:

- record the physical room (room scan) where the student takes the exam;

- $\quad \log /$ flag the incident or misbehaviour with a time-stamp, such that instructors can quickly navigate within the recording;

- $\quad$ artificial intelligence (AI) support;

- record the screen of the student;

- record webcam/microphone feed;

- real-time audio analysis;

- head/eye movements of the student;

- review student behaviour for a second time;

- geo-fencing, that is, the use of a global positioning system (GPS) or radio frequency identification (RFID) to define geographic boundaries where the exam is allowed to take place;

- $\quad$ second webcam support.

- Force completion of the exam: If a student's cheating behaviour is detected, the system forces the exam to end;

- Live chat support: Direct interaction with the teacher during the online exam;

- GDPR compliance: Acronym for general data protection regulation or general regulation for data protection, European Regulation No. 2016/679 on privacy;

- Free: This indicates a paid or free system;

- User-friendly: It is easy to use even for those who are not experts;

- Platforms: Compatibility with one or more of the following operating systems: Windows, Mac OS, and Linux for desktops, and Android and Apple iOS for mobile devices;

- Plugin/Browser extension: A particular Internet browser is requested to be installed, for example, Google Chrome;

- Needs client installation: This indicates that the system requires the user to install network client components in a parent directory on a client computer;

- Internet connection: Internet access is required;

- Open source: Software not protected by copyright and freely accessible and editable by users;

- Category: Proctoring categories to which the system belongs, that is, automated proctoring (AP), live proctoring (LP), or recorded proctoring (RP).

Short descriptions of the reviewed systems are described below:

1. ProctorU offers both automated and live proctoring. Prior to starting the exam, the students verify their identity by comparing their student ID card with a photo stored in the institution's database. ProctorU comes in three versions: The Live+ version flags and prevents suspicious behaviour through an immediate intervention by a human proctor. Review + and Record + use AI technologies to detect and flag suspicious events, which are revised after the end of the exam. A preliminary keystroke analysis monitors whether the same person is attending the session. ProctorU requires Chrome or Firefox extension to work (31-36).

2. Proctortrack offers a live and fully automated online remote proctoring service that continuously verifies the identity of online test-takers while detecting and deterring any misconduct. Proctortrack is fully integrated with all major learning management systems (LMSs) such as Moodle, Canvas, Sakai, Blackboard, edX, and Brightspace, and works even when Internet interruptions occur. Through facial recognition algorithms, the platform controls the student examination by monitoring student behaviour and detecting whether the student leaves the 
Table 1. List of features or functions offered by commercial OPL systems (Part 1)

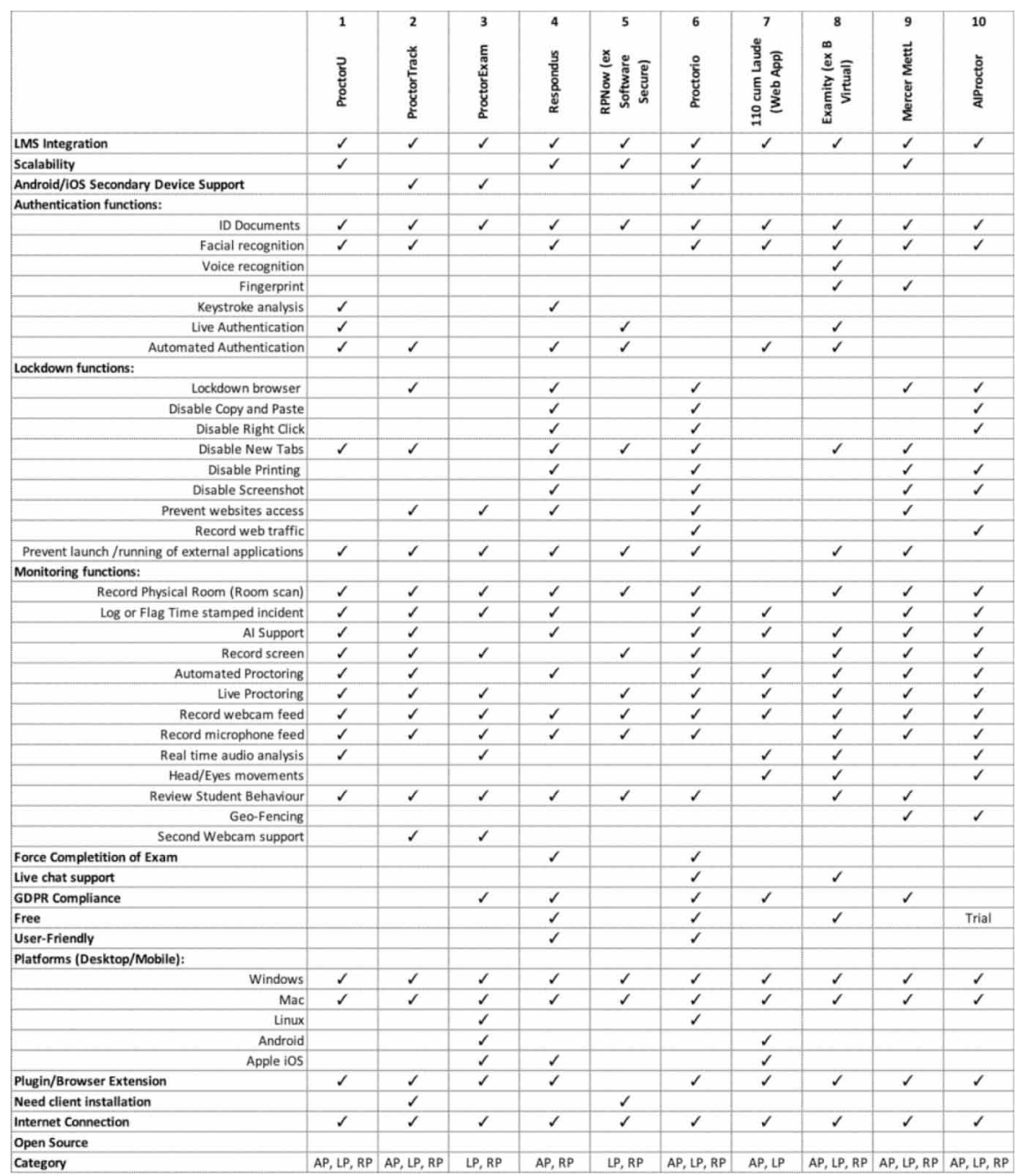

workplace, searches online for additional resources, or talks with another person, among other behaviours. The system verifies the student's identity through a multi-factor biometric analysis of the face characteristics, ID cards, and knuckle scans, which are compared with the student's baseline biometric profile, stored in a file. It also provides a mobile app that uses the front camera of the computer for facial and ID confirmation and for the other uses during the live exam (2-9).

3. ProctorExam is a cloud-based solution that is compliant with General Data Protection Regulation (GDPR) and is designed to run as stand-alone software or embedded within a learning management system. Its web-based infrastructure offers live proctoring and Record and Review solutions, supervision of test-takers with up to three simultaneous feeds such as screen-sharing, audio/ video webcams, and a secondary mobile camera, attributable to an Android or iOS mobile app on the candidates' smartphones or tablets. ProctorExam creates a $360^{\circ}$ view of the candidate workspace in such a way that the entire test session can be recorded from multiple angles. Lacking an AI support to detect misconduct, the teacher has a monitoring interface that displays all active candidates in a panel. A rotation between candidates takes place automatically every $7 \mathrm{~s}$, ensuring 
Table 2. List of features or functions offered by commercial OPL systems (Part 2)

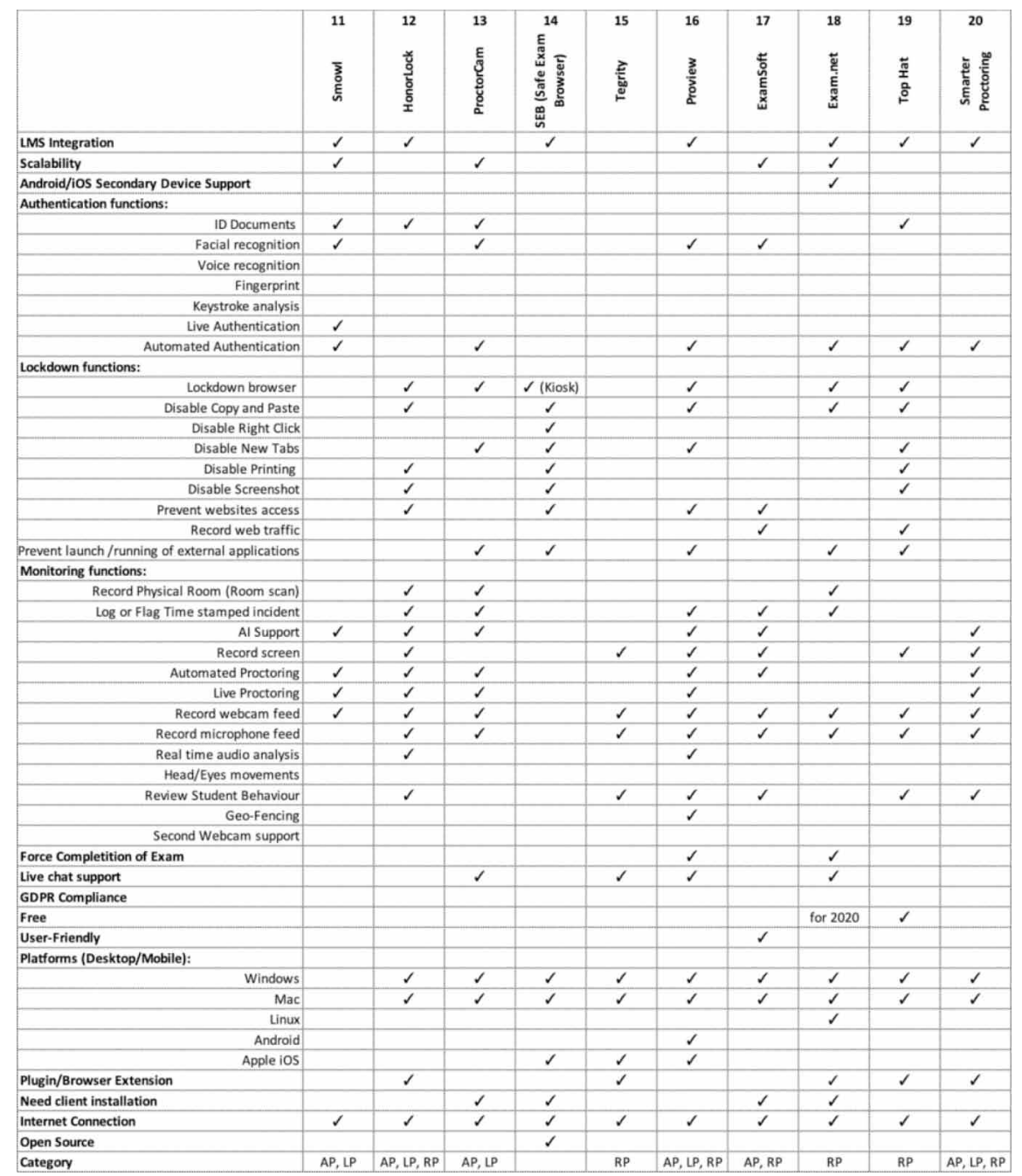

an equal inspection time among test-takers. At the end of the exam, the reviewer analyses the recorded video streams and students' browser activities, flagging the specific sessions that the reviewer considers anomalous and placing a time-stamp on the incidents (10-12).

4. Respondus provides a fully automated proctoring solution for online exams that can be integrated with many existing LMSs. The main module is the Respondus Monitor, which protects the exam questions by blocking the print functions, keyboard shortcuts, copy-and-paste, and screen-capture programs, accessing other applications, or visiting other websites. After the installation, the software executes an auto-launch from the student's browser for a start-up process that includes a webcam audio/video check. A pre-exam sequence is needed for identification and room scan. During the exam, the webcam records the student, and later, an artificial intelligence engine (Monitor AI) performs a video analysis to identify anomalous behaviours. After these operations, the system generates a report stored in the review priority portal, which ranks the proctoring results according to the risk of exam violations having occurred $(14,15)$. 
Table 3. List of features or functions offered by commercial OPL systems (Part 3)

\begin{tabular}{|c|c|c|c|c|c|c|c|c|c|}
\hline & 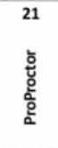 & 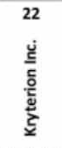 &  & 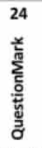 & 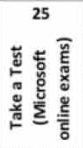 & 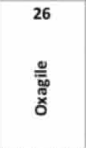 & 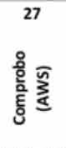 & 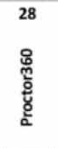 & 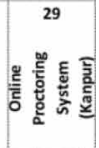 \\
\hline LMS Integration & & & & $\checkmark$ & & $\checkmark$ & $\checkmark$ & & \\
\hline Scalability & $\checkmark$ & $\checkmark$ & & $\checkmark$ & & $\checkmark$ & & & \\
\hline \multicolumn{10}{|l|}{ Android/iOS Secondary Device Support } \\
\hline \multicolumn{10}{|l|}{ Authentication functions: } \\
\hline ID Documents & $\checkmark$ & & $\checkmark$ & & & & $\checkmark$ & & \\
\hline Facial recognition & $\checkmark$ & & & & & $\checkmark$ & $\checkmark$ & & \\
\hline \multicolumn{10}{|l|}{ Voice recognition } \\
\hline \multicolumn{10}{|l|}{ Fingerprint } \\
\hline Keystroke analysis & & $\checkmark$ & & & & $\checkmark$ & & & \\
\hline Live Authentication & $\checkmark$ & $\checkmark$ & & & $\checkmark$ & $\checkmark$ & $\checkmark$ & & \\
\hline Automated Authentication & $\checkmark$ & $\checkmark$ & $\checkmark$ & $\checkmark$ & $\checkmark$ & $\checkmark$ & $\checkmark$ & & \\
\hline \multicolumn{10}{|l|}{ Lockdown functions: } \\
\hline Lockdown browser & $\checkmark$ & & & & & $\checkmark$ & $\checkmark$ & & \\
\hline Disable Copy and Paste & & $\checkmark$ & $\checkmark$ & & $\checkmark$ & & & & \\
\hline \multicolumn{10}{|l|}{ Disable Right Click } \\
\hline Disable New Tabs & & $\checkmark$ & $\checkmark$ & $\checkmark$ & $\checkmark$ & & & & \\
\hline Disable Printing & & $\checkmark$ & $\checkmark$ & & $\checkmark$ & & & & \\
\hline Disable Screenshot & & $\checkmark$ & $\checkmark$ & & $\checkmark$ & & & & \\
\hline Prevent websites access & & & & & $\checkmark$ & & $\checkmark$ & & \\
\hline \multicolumn{10}{|l|}{ Record web traffic } \\
\hline Prevent launch/running of external applications & & $\checkmark$ & $\checkmark$ & $\checkmark$ & $\checkmark$ & & $\checkmark$ & & \\
\hline \multicolumn{10}{|l|}{ Monitoring functions: } \\
\hline Record Physical Room (Room scan) & $\checkmark$ & & $\checkmark$ & $\checkmark$ & $\checkmark$ & & $\checkmark$ & $\checkmark$ & $\checkmark$ \\
\hline Log or Flag Time stamped incident & & & & & & $\checkmark$ & $\checkmark$ & & \\
\hline Al Support & $\checkmark$ & & & & & $\checkmark$ & $\checkmark$ & & \\
\hline Record screen & & & & $\checkmark$ & & $\checkmark$ & & & \\
\hline Automated Proctoring & & $\checkmark$ & & & $\checkmark$ & $\checkmark$ & $\checkmark$ & & $\checkmark$ \\
\hline Live Proctoring & $\checkmark$ & & $\checkmark$ & & & $\checkmark$ & & $\checkmark$ & \\
\hline Record webcam feed & $\checkmark$ & $\checkmark$ & $\checkmark$ & $\checkmark$ & $\checkmark$ & $\checkmark$ & $\checkmark$ & $\checkmark$ & $\checkmark$ \\
\hline Record microphone feed & $\checkmark$ & $\checkmark$ & $\checkmark$ & $\checkmark$ & $\checkmark$ & $\checkmark$ & $\checkmark$ & $\checkmark$ & $\checkmark$ \\
\hline Real time audio analysis & & & & & & $\checkmark$ & & & \\
\hline Head/Eyes movements & & & & & &  & $\checkmark$ & & \\
\hline Review Student Behaviour & $\checkmark$ & & & $\checkmark$ & & $\checkmark$ & & $\checkmark$ & $\checkmark$ \\
\hline Geo-Fencing & $\checkmark$ & & & & $\checkmark$ & & & & \\
\hline \multicolumn{10}{|l|}{ Second Webcam support } \\
\hline Force Completition of Exam & $\checkmark$ & & & $\checkmark$ & $\checkmark$ & & & & \\
\hline Live chat support & & $\checkmark$ & $\checkmark$ & & $\checkmark$ & $\checkmark$ & & & \\
\hline GDPR Compliance & & & & & & $\checkmark$ & $\checkmark$ & & \\
\hline Free & & & & & & & & & $\checkmark$ \\
\hline User-Friendly & & $\checkmark$ & & & & & & & \\
\hline \multicolumn{10}{|l|}{ Platforms (Desktop/Mobile): } \\
\hline Windows & $\checkmark$ & $\checkmark$ & $\checkmark$ & $\checkmark$ & $\checkmark$ & & & & \\
\hline Mac & $\checkmark$ & $\checkmark$ & $\checkmark$ & $\checkmark$ & $\checkmark$ & & & & \\
\hline \multicolumn{10}{|l|}{ Linux } \\
\hline Android & & & & & $\checkmark$ & & & & \\
\hline Apple iOS & & & & & $\checkmark$ & & & & \\
\hline Plugin/Browser Extension & $\checkmark$ & & & & & & & & \\
\hline Need client installation & $\checkmark$ & & & & & & & & \\
\hline Internet Connection & $\checkmark$ & $\checkmark$ & $\checkmark$ & $\checkmark$ & $\checkmark$ & $\checkmark$ & $\checkmark$ & $\checkmark$ & $\checkmark$ \\
\hline \multicolumn{10}{|l|}{ Open Source } \\
\hline Category & LP, RP & AP, RP & $L P$ & RP & AP & $A P, L P, R P$ & AP, RP & $L P, R P$ & $A P, R P$ \\
\hline
\end{tabular}

5. RPNow is a cloud-based platform that requires a client installation and works with any LMS supporting the Learning Tools Interoperability standard. With RPNow, the video/audio webcam and desktop data are streamed directly to the cloud. Before the exam, RPNow alerts the student if more than one monitor is being used or any prohibited programs are running, and provides a button to close them. It then proceeds with the identification procedure by taking a photograph of the student and a room scan. Human proctors then review the video and comment on any suspicious activities or rule violations, and based on their judgement, they provide a final exam analysis (16-18).

6. Proctorio allows live and automated authentication by employing machine learning for ID verification and facial detection. The program requires the Google Chrome web browser and a Proctorio plugin that enables webcam and microphone recording as well as browser lockdown 
features. This software disables many computer functions, including new Internet tabs, printing, use of the clipboard, and the right-click feature of the mouse. It prevents the test-taker from accessing other programs during the test and clears temporary Internet files to avoid retaining and redistributing the exam materials. Proctorio may ask for a room scan both at the beginning of the exam and in the case of suspicious noises. During the exam, Proctorio records the webcam video and audio, screens the examinee activities, and collects further information (e.g., the eyes, mouth, and head movements) to identify and flag anomalous behaviours, which are listed in a report for later review $(13,19-30)$.

7. 110 Cum Laude is an Italian web-based application developed and built on Morphocast neural network technology. The program monitors online exams and works in tandem with a few types of conferencing software such as Zoom, Microsoft Teams, Meet, or Cisco Webex. The system provides real-time analysis and alerts examiners by taking a picture of anomalous behaviour and sending immediate feedback to avoid post-exam reviews. A peculiar feature is the control of attention, emotion, and mood status of the examinee, to detect any expression of a student's psychological status. By using facial recognition biometric functions, the system continuously verifies the identity and presence of the examinees during the exam and detects their eyes, head movements, and position, as well as the presence of other persons within the workplace (53).

8. Examity is integrated with different LMSs, where students can create their personal profiles before starting the exam. The pre-exam step begins with a comprehensive auto-authentication in which the student submits an official ID and a real-time image taken from a webcam. In addition, the system requires students to answer a series of questions to further verify their identity and to submit a digital signature that can be used as a keystroke index. The ID, real-time image, and keystroke cadence are matched against the test-taker pre-exam recorded data. After authentication, the student downloads and installs the ExamiLock software, which allows the recording of the workplace and the beginning of the exam. The entire exam is monitored by AI algorithms and time stamps of possible incidents. The video is posted to the Examity dashboard for a review by the examiner (54-56).

9. MettL proctoring functions are authenticated through a photograph, ID card, or OTP. The AI-based proctoring procedure is designed to detect any malpractice live and to flag various anomalous cases, including the presence of mobile phones, multiple people, moments of distraction of the examinees, and missing form frames during the exam. The program calculates the likelihood of misconduct by defining a high, medium, and low level for cheating. MettL uses a MettL Secure Browser, which is a lockdown software for disabling new browser tabs, navigation through deny-listed websites, USB, email, virtual machines, or remote desktops. The material for the examination is also secured to avoid being printed, copied, or pasted to other devices. It is GDPR compliant and includes geo-fencing for the examinee (57-59).

10. AIProctor can be used with existing LMSs and provides an online testing platform for remote exams. It identifies and tracks anomalous student behaviour through an AI cheating detection device in collaboration with a human proctor. For the verification process, AIProctor captures the student face and ID and makes a $360^{\circ}$ room scan. AI algorithms track the head positions and movements, detect the possible presence of multiple persons during the exam, and flag anomalous movements or activities. The entire exam is recorded so that the examiner can review the video to find possible anomalies. In addition, human proctors can monitor live video and audio of the exam (60).

11. Smowl combines AI algorithms and human supervision to verify the identity of the examinee and to detect anomalous behaviours. It controls the student's behaviour without interacting with the student. It does not require additional software and can be used within a web browser. Smowl records the student's identity by capturing three photographs through the webcam, taking additional images of the student randomly during the exam, and matching them with the initial recorded pictures. All photographs are analysed and reviewed by an automated proctoring service 
that is constantly active during the exam. The results are then automatically given to the teacher in the LMS portal at the end of the exam (61).

12. ProctorCam consists of both live and automated proctoring systems. It uses AI to support live human proctoring, using a web monitoring tool that collects any indication of the test infringement and other anomalous activities. Before taking the test, the examinee needs to download and install the OnVUE application, which checks the hardware equipment of the student's computer, takes a photograph of the examinee and the student ID to verify the student's identity, records four photographs of the workplace, and shuts down other applications running on the computer during the exam. A human supervisor analyses the images of the student's room, and if the authentication process does not indicate any problems, the examiner is allowed to conduct the test. The examiner can flag anomalies and other testing-related incidents, and the exam can be interrupted if an illicit behaviour is detected. During the exam, if necessary, the examinee can use a live chat to contact the proctor $(37,62)$.

13. The Honorlock proctoring service works as a Google Chrome extension that disables copypaste and printing functions, preventing the use of multiple monitors and access to materials stored on the computer. Before starting the exam, the system records the student's face and ID for authentication and creates a 360-degree room scan. During the exam, the system records the screen, audio/video, and web activities of the student's computer. AI algorithms detect the presence of extraneous voices and the use of secondary devices such as smartphones, tablets or laptops. In addition, a live proctor pop-out during the exam is shown if the student conducts anomalous activities, with the purpose of saving a substantial amount of time in reviewing reports or video sequences (63-67).

14. Safe Exam Browser (SEB) is an open-source application developed to provide a "safe environment" that allows students to take the exam at home with their own device. SEB is currently available only for Windows and Mac, and contains several technologies to prevent examinees from obtaining access to forbidden materials or software ${ }^{1}(50)$.

15. Tegrity does not include lockdown functions, but records audio, video, and screen inputs from the student's computer, which can provide information about the events that occurred during the exam (49).

16. Proview provides both live and automated proctoring, student identity authentication (using a 2-factor authentication method), and integration with existing LMSs or websites that need to be proctored. It is designed to also work with low-bandwidth Internet connections. It has browser policing and real-time alerts. The system prevents misconduct by forbidding examinees to copy and paste answers, checking their browser navigation, capturing screens, monitoring real-time student activities, and recording video. An app is also available for iOS and Android. Direct communication through the chat box between the examiner and the examinee is provided. Geo-fencing functionality is also available. AI support consists of face or voice recognition for detecting anomalous activities (48).

17. ExamSoft locks down the student's device and blocks Wi-Fi connections completely. The student begins the exam by logging into the identity verification procedure through a two-step authentication process. After the commencement of the exam, ExamMonitor records audio and video of the examinee behaviours. These records create a log of all of examinee actions, and the AI software then identifies the anomalies in these actions, such as unusual body or eye movements, and among the background noise, shows any "red flags" concomitantly based on these anomalies (47).

18. Exam.net is available for free from 2020 outside Sweden. It works on every browser and PC/ tablet and on different operating systems. Exam.net can be used together with several video conference programs to control student activities (lockdown mode, cheat log, text evolvement, copy/paste blocking), while the recorded video shows what happens at the student's workplace (46). 
19. Top Hat is available both for computers and mobile devices. It provides ID verification and analyses student activities using advanced facial detection functions and AI, generating a report that flags student anomalies. It has a set of lockdown features, that is, disabling printing, disabling the sharing of materials, screen capturing, blocking downloads, monitoring the examinee's web traffic, blocking the opening of browser tabs, and preventing screenshot capturing $(19,45)$.

20. SmarterProctoring is a proctoring platform that relies on its services on ProctorU systems. It integrates with the major LMSs and offers all types of proctoring modalities, including automated and in-person functions. This platform is designed to schedule and deliver proctored exams and allows choosing which proctoring options will be available during each session. To use SmarterProctoring, the computer must have JavaScript and cookies enabled $(43,44)$.

21. ProProctor is an application that must be downloaded by the examinee. ProProctor works only on Windows and Mac. Google Chrome is necessary as a web browser. Recording and reviewing functions are available. Before the exam, the examinee's identity (name, address, exam details, valid identity document with a photograph, and signature) is checked. The examinee can speak with and/or send a message to the examiner if help is needed during the exam. In addition, an environmental check is performed before starting the exam, and the workplace plus the surrounding area are continuously monitored through a webcam and microphones. The exam is interrupted when a violation of the rules is detected (42).

22. Kryterion, Inc. requires the installation of two apps: Sentinel and Biometrics. Sentinel analyses the typing style of the examinee on the keyboard. The workplace must have an uncluttered background, good overhead lighting, and a good Internet connection. The student must be alone in the room. Surfing on the Internet or opening additional browser windows is not allowed (41).

23. Loyalist Exam Services offer a live proctoring service. The system requires a desktop computer or laptop, a working built-in or external webcam and microphone, a high-speed Internet connection, and a browser with pop-up blockers disabled. The proctoring system analyses an examinee's movements and noise throughout the entire duration of the exam. The proctor needs permission to control the examinee's computer, check the examinee's photo identification, and inspect the room. All exams are recorded to ensure and enforce compliance from the examinees. The examinee can use only one screen during the exam (39).

24. QuestionMark works only on Windows and Mac computers and can be integrated with existing LMSs. Forbidden applications, such as Outlook, Skype, and GoToMeeting, must be closed before the exam. The program can record the computer desktop. It is usually used in connection with videoconferencing applications. No phone, no headset, or dual monitors are allowed. Webcams, speakers, and microphones must be turned on throughout the test because the proctoring program continuously analyses the examinee's behaviour. QuestionMark provides audio/video recordings of the entire exam for a possible later review in the case of irregularities. Before starting the test, the program verifies the identity of the examinee. During the test, the proctor can intervene, forcing the completion of the exam if an anomalous behaviour is detected (40).

25. The Take a Test app locks the PC and displays a web page for conducting online exams. The Tests and Exams app makes the browser "safe" by preventing students from using other Internet resources during the test, showing only the exam questions, deleting the contents of the notes, disabling websites, opening or accessing other apps, disabling screen sharing, printing and taking a screenshot, providing text suggestions (such as the use of autofill features), and using Cortana. The student's desktop is locked during the exam, and the screen is captured by the system (38).

26. Oxagile integrates with LMSs, enterprise learning systems, and eLearning portals. It uses AIbased analyses to supervise test-takers using webcam, microphone, and screen access and involves live proctors to review cases of illicit behaviour. It works with a lockdown browser that disables several computer capabilities of the student and allows a fully automated user registration with an identity verification procedure applied throughout the entire exam. AI algorithms analyse the end-to-end video recordings and flag any sign of suspicious behaviour by sending alerts to 
the teacher in real time. AI features regard face and emotional recognition, the light level in the workplace, the distance of the examinee from the camera, and head movements. In addition, Oxagile is GDPR compliant (68).

27. Comprobo is an automated proctoring service provider that can be integrated with existing LMSs. It records the user's ID photograph before the exam and compares the image with a reference photograph. Alternatively, the examiner can directly verify the identity of the student. The platform allows the recording of the examinee's workplace and constantly checks that the person who started the exam does not change during the exam. The program flags anomalous movements or activities. In addition, AI algorithms implement the monitoring of biometric features by controlling whether the examinee is looking at the monitor screen without attempting to move away from the frame. The Comprobo system does not require any installation or browser extension software to work and is GDPR compliant (69).

28. Proctor 360 offers a live proctoring service and allows the monitoring of student activities in real time. Proctor360 uses a patented 360-degree testing cam, which is shipped to the student's home when the student signs up to take the exam. The student's identity is verified before the exam and the proctoring program is able to record a complete view of the workplace during the entire exam. When the exam is completed, the student must return the headset. Proctor360 archives a 360-degree video of the entire exam for later review, if necessary. To the best of our knowledge, this is the only system that exploits 360-degree cameras for remote proctoring (51).

29. Finally, the Kanpur project, available on GitHub, provides an automated online exam proctoring system with visual-audio sensors to detect cheating or illicit behaviour. The system monitors the room using two cameras and a microphone. The first camera is the webcam of the laptop itself. The other camera can be a smartphone camera. The built-in microphone of the laptop is used for voice recognition (52).

\section{MULTIDIMENSIONAL SCALING ANALYSIS OF OLP PROGRAMS}

We performed a multidimensional scaling (MDS) analysis to analyse how the different proctoring programs are grouped based on the services they offer. MDS is a statistical technique used to visualise the level of similarity of individual cases of a dataset. MDS is used to transform information regarding the pairwise distances among a set of $\mathrm{n}$ objects or individuals into a configuration of $\mathrm{n}$ points mapped into an abstract Cartesian space (Mead, 1992). Pairwise distances can be estimated using qualitative or quantitative data (Mead, 1992).

The proctoring systems used for the analysis are listed in Tables 1-3. We considered the three principal services offered by the programs, which are articulated based on numerous characteristics or functions, i.e. for identifying the examinee, for a computer lockdown, and for monitor settings. The identification functions were document identification, facial recognition, voice recognition, fingerprints, keystroke analysis, live authentication, and automated authentication. The lockdown functions were a browser lockdown, disabling copy and paste, disabling a right click, disabling the use of new tabs, disabling printing, disabling screenshots, preventing website access, recording web traffic, and preventing launching and running of external applications. The monitor setting functions were a room scan, log or flagging of time-stamped incidents, AI support, screen recording, automated proctoring, live proctoring, webcam feed recording, microphone feed recording, real-time audio analysis, head and eye-movements, a review of student behaviours, geo-fencing, and second webcam support. MDS grouped the proctoring programs based on the presence or absence of these functions. For example, if two programs having the same functions are grouped together, and one of them has some functions and the other has completely different functions, they are divided into different groups.

Figure 1 shows four principal groups detected using MDS. Group 3 is composed of proctoring programs that do not have functions for identification (SEB, Tegrity, Proctor360, or Online Proctoring India). Group 4 is composed of programs that offer one to three functions (ProctorExam, HonorLock, 
Figure 1. MDS grouping based on the number of identification methods offered by the proctoring programs



ExamSoft, Loyalist Exam Services, Top Hat, Proctorio, AIProctor, or Mercer MettL) principally based on document identification and automated authentication. Group 1 is composed of programs that offer one to four identification functions (Exam net, SmarterProctoring, QuestionMark, Proview, Take a Test app, Kryterion, Inc., RPNow, or Oxagile) principally based on live authentication and automated authentication. Group 2 is composed of programs that offer three to seven identification functions (ProctorTrack, 110 cum Laude, ProctorCam Pearson VUE, Respondus, Smowl, ProProctor, Comprobo AWS, ProctorU, or Examity). The programs that offer the highest number of identification functions are ProctorU (five functions) and Examity (six functions). The most frequent functions offered by programs are document identification (17 programs), facial recognition (15 programs), and automated authentication (19 programs). The least frequent functions are a keystroke analysis (four programs) and a fingerprint (two programs).

Figure 2 shows five principal groups detected using MDS. Group 1 is composed of programs that do not offer lockdown functions (110 cum Laude, Smowl, Tegrity, SmarterProctoring, Proctor360, or Online Proctoring India) or offer from one to two lockdown functions (ProctorCam Pearson VUE,

Figure 2. MDS grouping based on the number of lockdown methods offered by proctoring programs

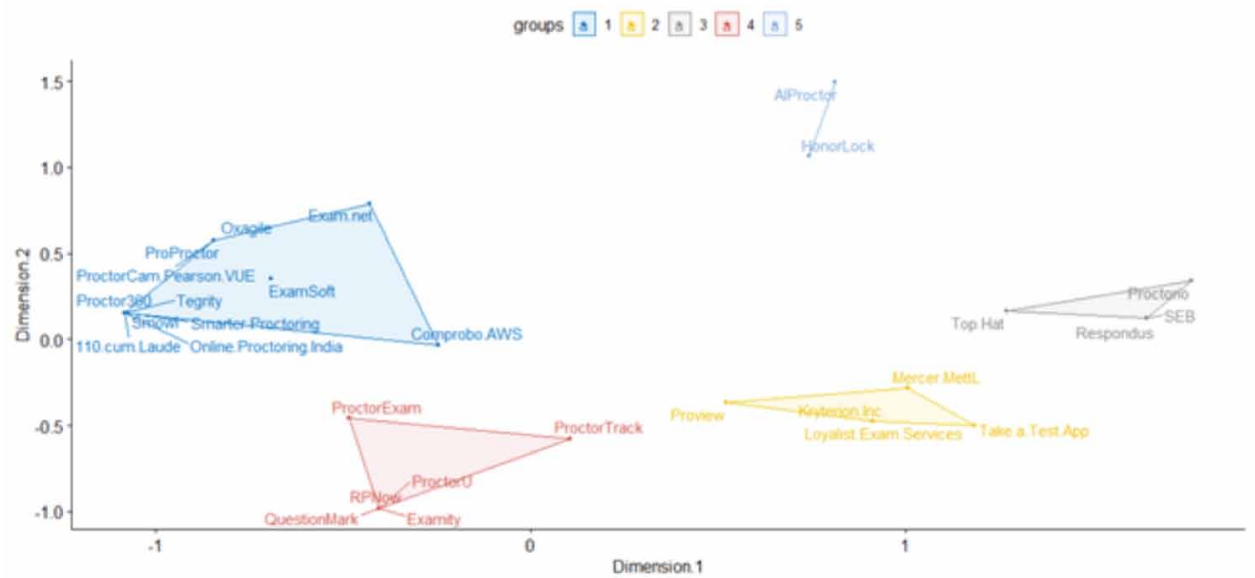


Figure 3. MDS grouping based on the number of monitor setting methods that are offered by proctoring programs



ProProctor, Oxagile, ExamSoft, Exam net, or Comprobo AWS) principally based on a browser lockdown. Group 4 is composed of programs that offer two (ProctorU, QuestionMark, ProctorExam, RPNow, or Examity) or four (ProctorTrack) lockdown functions principally based on preventing the launching and running of external applications. Group 2 is composed of programs that offer four to six lockdown functions (Kryterion Inc., Loyalist Exam Services, Mercer MettL, Take a Test App, or Proview) principally based on disabling new tabs, printing, and taking a screenshot. Group 5 is composed of programs that offer five to six lockdown functions (HonorLock, AIProctor) principally based on disabling a copy and paste. Group 3 is composed of programs offering the highest number (from seven to nine) of lockdown functions (Top Hat, Respondus, SEB, or Proctorio). The programs with the highest number of lockdown functions are Respondus and SEB (eight functions) and Proctorio (nine functions). The most frequent functions offered by programs are preventing the launching and running of external applications (16 programs), browser lockdown (14 programs), and disabling new tabs (14 programs). The least frequent functions are disabling a right click (four programs) and recording web traffic (four programs).

Figure 3 shows five principal groups detected using MDS. Group 1 is composed of programs that do not have functions for monitoring settings (SEB) or from three (Kryterion Inc., Exam net, Proctor360, Loyalist Exam Services) to five functions (Take a Test app), principally based on webcam and microphone feed recording. Group 2 is composed of programs that offer four to six monitoring functions (Tegrity, Top Hat, Online Proctoring India, QuestionMark, or RPNow), principally based on recording the screen and reviewing student behaviour. Group 3 is composed of programs that offer four to seven monitoring functions (Smowl, ProctorCam Pearson VUE, or 110 cum Laude), principally based on AI support and Automated Proctoring. Group 4 is composed of programs that offer six to eight monitoring functions (Comprobo AWS, Respondus, ExamSoft, SmarterProctoring, ProProctor, ProctorTrack, and ProctorExam), principally based on a room scan and log or flagging of a time-stamped incident. Group 5 is composed of programs (Proctorio, Proview, ProctorU, Examity, Mercer MettL, HonorLock, Oxagile, or AIProctor) that have the highest number of monitoring functions (from 9 to 11). The programs with the higher number of monitoring functions are ProctorU, Examity, Mercer MettL, HonorLock, and Oxagile, all with 10 functions, and AIProctor (11 functions). The most frequently offered functions are webcam feed recording (28 programs), microphone feed recording (25 programs), and a review of student behaviours (19 programs). The least are head and eye movements (five programs), Geo-Fencing (five programs), and a second webcam support (two programs). 
The results of the MDS analysis, with programs clustered into four or five groups, demonstrated a high variability between proctoring program characteristics. Some programs constantly show a high or low number of characteristics for each dimension (identification methods, lockdown methods, and monitor settings). For example, ProctorCam Pearson VUE has a few characteristics in each dimension, whereas Respondus and Proctorio continuously have many characteristics. Other programs, however, can have a few functions in one dimension and many in others (e.g. SEB, 110 cum laude, and HonorLock). In some programs, there is generally a wide or narrow offering of functions, whereas in other programs, some characteristics were developed for a specific dimension but not for others. Therefore, there is extremely high variability in the functions offered by proctoring systems.

\section{CASE STUDIES}

The section reports two case studies in two different courses: The first regards exams conducted using a commercial proctoring system, whereas the second discusses the experience with a manual proctoring approach. The two systems were chosen by different institutions, as the result of a political decision that took into account both the available budget and previous experience with the system. The two case studies complement the previous analyses, which are based on features promoted by venders, by discussing the pros and cons of two different approaches in two real situations.

\section{Use of a Proctoring System in a University Course of the Psychology Faculty: Automated Commercial Proctoring}

Some of the authors used a proctoring program ${ }^{2}$ for conducting online exams of two courses: "Theory and techniques of psychological testing" and "Methodology for psychological research." In the first course, 54 students participated in the exam, whereas in the second, 26 students participated. The exam session lasted $60 \mathrm{~min}$. For each session, two instructors were involved. The proctoring program first took a photograph of the student and the student's identity card, and the program then asked the student to make a video of the environment to check the presence of other persons in the room and whether the desktop was clean. After this operation, the exam started. At the time of the examination, the program recorded all head and eye movements of the student and all environment noises. At the end of the exam, all video clips were stored on the proctoring program website. Video sequences were automatically classified by the proctoring program and ordered in relation to their priority. The priority indicated the presence of problems during the exam. The priority levels were high, medium, and low. Table 4 shows the number of video clips with the three priority levels and with the number of video clips for which there were recording problems independent of student activities during the session.

Only a few cases had anomalies in student activities during the exam (a case with high or medium priority). In the Theory and techniques of psychological testing, only $5.6 \%$ of the video clips had high or medium priority, and in Methodology for psychological research, 3.8\% of the video clips had high or medium priority. However, after a visual analysis of the content of all video clips to find the behaviour anomalies in students, many more cases of anomalous activities were found by the

Table 4. Frequency of high, medium, and low priority results and number of recording problems for each exam. Percentages are shown in parentheses.

\begin{tabular}{|c|c|c|c|c|c|}
\hline Exam & $\begin{array}{c}\text { High } \\
\text { Priority }\end{array}$ & $\begin{array}{c}\text { Medium } \\
\text { Priority }\end{array}$ & $\begin{array}{c}\text { Low } \\
\text { Priority }\end{array}$ & $\begin{array}{c}\text { Recording } \\
\text { Problems }\end{array}$ & Totals \\
\hline $\begin{array}{c}\text { Theory and techniques of } \\
\text { psychological testing }\end{array}$ & $1(1.9 \%)$ & $2(3.7 \%)$ & $48(88.9 \%)$ & $3(5.6 \%)$ & $54(100 \%)$ \\
\hline $\begin{array}{c}\text { Methodology for psychological } \\
\text { research }\end{array}$ & $1(3.8 \%)$ & $0(0.0 \%)$ & $24(92.3 \%)$ & $1(3.8 \%)$ & $26(100 \%)$ \\
\hline
\end{tabular}


authors. The required time to analyse all video clips overall was approximately $23 \mathrm{~h}$ and $35 \mathrm{~min}$. In particular, for Theory and techniques, the authors found 13 video clips with anomalous activities (24.1\%), whereas for Methodology, the authors found three video clips (11.5\%). The difference between the frequencies of anomalous video clips found by the program and those found by visual inspection was significant for Theory and techniques (chi-square $=12.5 ; \mathrm{p}<.001$ ) and insignificant for Methodology (chi-square $=2.0 ; \mathrm{p}=.157$ ). Considering the level of priority by which the video clips were ordered, we found that for Theory and techniques, only 4 video clips showed anomalies after a visual inspection, which were in the first 27 positions. The remaining 9 video clips were in the last 27 positions. For Methodology, one of the anomalous video clips corresponded to the video with high priority, whereas the other two were at the 10th and 21st positions of priority. In summary, there was no good correspondence between the order of priority defined by the program and the behaviour of the anomalies identified through a visual inspection of the same video clips. This means that it is necessary to visually inspect all video clips to identify situations in which there are behavioural anomalies of the students. In addition, the order of priority does not help in clarifying dubious situations, which are always solved with a careful inspection of the student's head and eye movements and by listening carefully to the sounds recorded in the environment during the session. For example, for one of the students, there was a window behind the student, and from the reflection of this window, it was possible to detect the presence of another person in the room. However, according to the program, there were no anomalies in the video. In conclusion, many more possible situations and contingencies must be considered during an exam to further improve the capacity of proctoring programs to find anomalies in student activities and to help examiners efficiently find anomalies in the students' performances.

\section{The Course of Information Processing Systems: Live Human Proctoring}

The course of Information processing systems in the master's degree courses of Nursing Sciences and Prevention Sciences at the University of L'Aquila (Italy), includes 92 students, and focuses on the statistical analysis of health data. To pass the course, students have to complete a practical assignment and obtain a score of at least 18 over a maximum of 30 points (possibly "cum laude"). Before the COVID-19 outbreak, the students had to complete the assignment using a computer in a dedicated room at the University under the surveillance of the teacher. After the lockdown, there was a long debate on how to re-organise all courses of the university. In the end, the university opted for a proprietary videoconferencing platform to support both the lectures and the exams, and defined several guidelines describing how to perform them through the videoconferencing platform. With regard to the course under discussion, these decisions required the teachers to reorganise the evaluation activity as follows.

Each student had to use a computer at home and a smartphone/tablet with an active microphone, framing both the computer (in particular, the monitor) and the student from a lateral viewpoint. The exam must be manually proctored by two professors using only the videoconferencing platform to allow them to identify the student, ask the student to show the room and desk, ask the student to place the smartphone/tablet in the proper position, monitor the student behaviours by listening and observing the different framings, and reschedule the exam in case of persistent network problems. The possibility of using an automated proctoring system was discouraged.

The student identification phase proceeded with few issues. Most of the students were already registered on the virtual secretary (an online platform used by the administrative offices). Only some of them were not registered with a photo and had to show their ID card by using the webcam. In a few cases, the connection was so poor that it was impossible to read the personal information or to recognise the student's face.

The preparation phase (that is, room control and proper positioning of the smartphone) was confusing in some cases, given the need to place the smartphone in a position enabling the monitoring 
of the student behaviour, as well as due to the logistic difficulties of the students, such as the lack of smartphone support and lights coming from the windows that prevented viewing the computer screen.

Figure 4 shows a sample exam, in the surveillance phase, during which a professor simultaneously monitored the behaviour of two students.

Monitoring was quite difficult. One of the most challenging issues was audio. The students were required to keep their microphone on (to enable the professor to listen to any possible suggestions), but occasionally strong external noises occurred (e.g., trucks), or annoying echoes were generated because of a poor network connection or because the students kept the videoconferencing platform open on both the computer and the smartphone/tablet. Nevertheless, the actual weakness of this proctoring method was the difficulty of viewing both the student's behaviours and the computer monitor clearly and simultaneously (e.g., to spot the use of messaging systems or any other type of misconduct). Logistic and network issues made proctoring extremely challenging. In addition, the lack of a native or automated support in the videoconference system for switching from one participant to another made the surveillance activity extremely intricate. Furthermore, in only one case out of a total of 149 exams $\left(0.67 \%, \mathrm{IC}_{95 \%}[0.02 \%, 3.68 \%]\right)$, owing to persistent network issues, the professors had to interrupt the exam of the student and reschedule it in a different time slot. Each exam session included no more than 20 students at the same time to reduce the network load and enable the instructors to monitor the students' behaviours. On an average, each exam session lasted approximately $1 \mathrm{~h}$ and 30 $\mathrm{min}$, that is, $1 \mathrm{~h}$ to complete the assignment, and $30 \mathrm{~min}$ for the identification and preparation phases.

However, this method enabled the professors to conduct the exam for a vision-impaired student, who needed support to check the formulas (that is, the professors had to re-read the formulas to enable the student to correct them, if wrong), and to find the proper buttons on the software interface. In this case, the videoconferencing platform offered sufficient flexibility to enable both the professors to help the student and the student herself to complete the exam without issues.

\section{Summary}

In summary, by dividing the phases of the online assessment into (i) pre-exam, (ii) monitoring, and (iii) post-exam activities, we found the following:

Figure 4. Sample exam

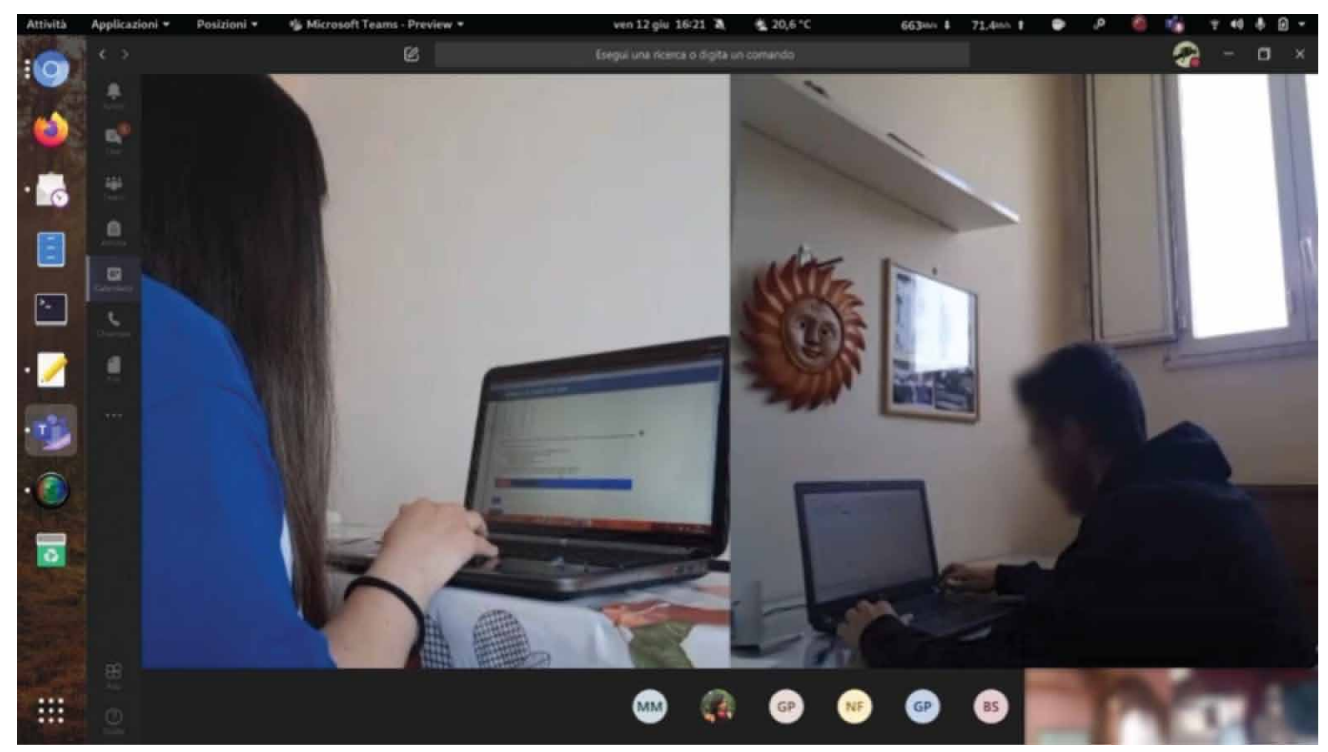


- Pre-Exam Activities: In the case of the automated OLP system, the teacher spent considerable time setting up the exam and proctoring options in the web portal, which needed adjustments related to the number of candidates, type of exam, and topics covered. For the manual case, the additional effort was limited to creating the event on the videoconferencing platform and inviting the students, who were organised into groups. Furthermore, the automated proctor had several problems with the student identification because many photo IDs and headshots were missing or out of focus, thus preventing correct student recognition, and with the automated room checks. By contrast, in the case of manual proctoring, the organisational effort made by the administrative staff (i.e., loading most of the ID cards into the virtual secretary) reduced the time required for verification and the problems in recognising the faces and personal information. However, asking the student to show the room, then the desk, then placing the smartphone/tablet in the proper position, required on average more than 1 min per student with the unpleasant feeling of entering a private space.

- Monitoring Activities: The surveillance phase took place automatically with an automated OLP system. Nevertheless, the students who used the automated proctor also reported difficulties owing to system crashes, general slowdowns, and computer issues related to the network connection speed, which caused unexpected disconnections or delays in the exam submission. In the case of manual proctoring, switching from one student to another was quite intricate because the videoconferencing platform did not provide a native way for switching from one student to another. Moreover, audio/video problems were found to be annoying, and in one case, the low quality of the Internet connection required rescheduling the exam.

- Post-Exam Activities: The automated OLP system required a significant amount of time after the exams, with the video clips having to be reviewed owing to a low precision in detecting suspicious activities. The proctoring system has been unable to detect and flag several types of misconduct such as the use of a smartphones, headphones, or learning materials, the presence of other people in the exam environment, and head movements. Therefore, in many cases, the teacher had to review a large majority of the exam video clips to manually identify students indulging in malpractice. However, no additional effort was required by the professors in the case of the manual proctoring approach.

\section{CONCLUSION}

During the analysis of commercial OLP systems, the information we collected from websites suggests that many systems integrate innovative technologies and features that give them the appearance to be effective in helping professors perform online exams. In this study, we initially identified the functions offered by such available programs to allow a comparison among them. The analysis of the OLP functions shows that there is significant variability between proctoring programs. Reasonably, this variability reflects the ongoing development of these programs owing to their high utility when students cannot have direct access to their educational institutions, not only owing to the current pandemic but also for other situations that may prevent the use of physical spaces (e.g., earthquakes) or for rational choices (e.g., online education). However, such variability can make it difficult for educators or teachers to find the best proctoring programs for conducting their exams or assessment trials.

Furthermore, we presented two case studies regarding the application of these systems under two real situations. In the first case, a commercial automated OLP system was used for conducting online exams of two university courses; in the second, a manual proctoring approach was used to apply the exams. The objective of this report is to offer a qualitative and concrete experience regarding the pros and cons of both approaches, thus complementing the analysis of commercial OLP systems.

In summary, the options offered by the proctoring systems are various, with many types of technological features. Our experience in the use of an automated OLP system has raised many doubts about the real support that proctoring systems can offer owing to several relevant problematic 
issues that emerged during their application. In particular, we observed a significant expenditure in the post-exam evaluation when a commercial proctoring system was used, whereas a higher time expenditure was observed during pre-exam operations when manual proctoring was applied. Furthermore, although the commercial OLP system showed extreme usefulness in automating the student identification, conducting a room scan, and monitoring the student performance, the anomalies detected in the students' behaviour did not correspond to those detected through a visual inspection of the video clips made by the examiner. The reasons behind these mistakes may be caused by the fact that many of the students did not have a good Internet connection, and thus the poor quality of the images prevented an accurate classification. Another possibility is the unexpected number of video clips to be processed owing to virtual learning and assessment. However, the poor precision in the automated classification of possible misconduct was quite disappointing.

Accordingly, we suggest the following for instructors interested in the use of an OLP program:

- Organisational effort from the administrative staff (e.g. in loading photos that can be used to easily recognise the students' faces, either automatically or manually) is mandatory.

- An automated OLP can be used to support manual proctoring, that is, as a live signalling tool for possible incidents to be reviewed in real-time by the human proctor.

- The videoconferencing approach is discouraged in the case of a large number of students, given the need to limit the number of students per session, the additional time to complete the pre-exam activities, and the intricacies of the monitoring.

Finally, given the problems of automated OLP in detecting misconduct, further research is mandatory and represents a crucial challenge for improving the quality of the current automated proctoring systems. 


\section{REFERENCES}

Alessio, H. M., Malay, N., Maurer, K., Bailer, A. J., \& Rubin, B. (2017). Examining the effect of proctoring on online test scores. Online Learning, 21(1), 146-161. doi:10.24059/olj.v21i1.885

Davis, A. B., Rand, R., \& Seay, R. (2016). Remote proctoring: The Effect of Proctoring on Grades. In Advances in Accounting Education: Teaching and Curriculum Innovations (Advances in Accounting Education, Vol. 18). Emerald Group Publishing Limited.

Foster, D., \& Layman, H. (2013). Online proctoring systems compared. Caveon Webinar Series. Retrieved from https://www.slideshare.net/caveonweb/caveon-webinar-series-online-proctoring-best-practices-oct-2013slideshare-final

Hylton, K., Levy, Y., \& Dringus, L. P. (2016). Utilizing webcam-based proctoring to deter misconduct in online exams. Computers \& Education, 92, 53-63. doi:10.1016/j.compedu.2015.10.002

Kolski, T., \& Weible, J. (2018). Examining the relationship between student test anxiety and webcam-based exam proctoring. Online Journal of Distance Learning Administration, 21(3).

Mead, A. (1992). Review of the development of multidimensional scaling methods. The Statistician, 41(1), 27-39. doi:10.2307/2348634

Milone, A. S., Cortese, A. M., Balestrieri, R. L., \& Pittenger, A. L. (2017). The impact of proctored online exams on the educational experience. Currents in Pharmacy Teaching \& Learning, 9(1), 108-114. doi:10.1016/j. cptl.2016.08.037 PMID:29180142

Okada, A., Scott, P., \& Mendona, M. (2015). Effective web videoconferencing for proctoring online oral exams: A case study at scale in Brazil. Open Praxis, 7(3), 227-242. doi:10.5944/openpraxis.7.3.215

O'Reilly, G., \& Creagh, J. (2015). Does the Shift to Cloud Delivery of Courses Compromise Quality Control. Higher Education in Transformation Conference, Dublin, Ireland.

O'Reilly, G., \& Creagh, J. (2016). A categorization of Online Proctoring. In Proceedings of Global Learn-Global Conference on Learning and Technology, (pp. 542-552). Limerick, Ireland: Association for the Advancement of Computing in Education (AACE). Retrieved from https://www.learntechlib.org/primary/p/172801/

Schulson, M. (2014). How to prevent online learning from ruining your reputation. eCampus News. https:// www.ecampusnews.com/2014/06/25/online-learning-proctoring-296/

Shingal, T. (2020). Online Test Proctoring: Comparing Online Proctoring Systems. Mettl blog article. https:// blog.mettl.com/online-test-proctored-exam/

Weiner, J. A., \& Hurtz, G. M. (2017). A comparative study of online remote proctored versus onsite proctored high-stakes exams. Journal of Applied Testing Technology, 18, 13-20.

\section{ENDNOTES}

Because Safe Exam Browser does not monitor or record audio/video during the exam sessions, it cannot be classified in any of the proctoring categories.

2 The name of the system is not reported to avoid any possible commercial conflict between the authors and the companies involved. The automated OLP system is one of the leading platforms, with features including automated identification of the test-takers, room scan, and misconduct. 


\section{WEBLIOGRAPHY}

https://en.unesco.org/covid19/educationresponse https://online.rowan.edu/proctoring https://www.proctortrack.com/ https://tlt.rutgers.edu/instructional-technology-tools/proctortrack https://www.ohio.edu/oit/services/testing/online-proctoring/help/instructor-tips

https://verificient.freshdesk.com/support/solutions/articles/1000281763-minimum-requirement-forproctortrack-on-mobile-

https://verificient.freshdesk.com/support/solutions/articles/1000281766-minimum-requirement-forlive-proctored-exams-

https://verificient.freshdesk.com/support/solutions/articles/1000065470-what-equipments-arerequired-to-use-proctortrack-

https://verificient.freshdesk.com/support/solutions/articles/1000141417-what-technical-requirementsdo-i-need-

https://proctorexam.com/ https://chrome.google.com/webstore/detail/proctorexam-screen-sharin/

digojkgonhgmnohbapdfjllpnmjmdhpg https://www.learninglight.com/online-proctoring-multi-layered-security/ https://www.depts.ttu.edu/elearning/blackboard/proctorio/proctorio-faculty-faqs.php https://web.respondus.com https://bloomuimdc.freshdesk.com/support/solutions/articles/35000045859-my-instructor-isrequiring-me-to-use-respondus-lockdown-browser-what-is-thishttps://www.psionline.com/ https://content.psionline.com/hubfs/Education\%20Client\%20Services/RPNow_4.0_features_and_ functionality.pdf?_ga=2.198658604.2048279474.1595074300-772636487.1595074300 https://support.edx.org/hc/en-us/articles/207249428-How-do-proctored-exams-workhttps://tophat.com/press-releases/top-hat-partners-with-proctorio/ https://proctorio.com https://www.depts.ttu.edu/elearning/blackboard/proctorio/proctorio-student-faq.php https://it.umn.edu/services-technologies/how-tos/proctorio-student-guide https://teach.uiowa.edu/respondus-vs-proctorio-comparison http://www.laspositascollege.edu/onlinelearning/online_services/online_proctoring.php https://www2.palomar.edu/pages/atrc/2018/02/06/brief-introduction-proctorio/ https://www.csuchico.edu/tlp/proctorio/student.shtml https://resourcecenter.odee.osu.edu/carmencanvas/proctorio-lock-down-options https://cbc.instructure.com/courses/1590941/pages/overview-of-proctorio https://cirrus.zendesk.com/hc/en-us/articles/360012590719-Automated-proctoring-by-Proctorio https://nursing.uw.edu/wp-content/uploads/2016/09/Proctorio-Info-FAQs.pdf?1477189052 https://www.proctoru.com/ https://teachonline.ca/tools-trends/tools-trends/best-practices-around-world-including-ontario/ teaching-and-learning/international-resources-and-services-teaching-and-learning/secure-onlineproctoring https://smarterproctoring.zendesk.com/hc/en-us/articles/360021230192-Scheduling-an-Online-

Proctoring-Session-ProctorU

https://my.uq.edu.au/information-and-services/student-support/proctoru-faq https://www.southern.edu/academics/academic-sites/online-campus/proctoring.html https://online.illinois.edu/proctoru http://content.nremt.org/static/documents/About\%20OnVUE.pdf 
https://docs.microsoft.com/it-it/education/windows/take-tests-in-windows-10

http://loyalistexamservices.com/

https://www.questionmark.com/platform-services/proctoring/

https://www.kryteriononline.com/

https://www.prometric.com/it/procurement-remoto

https://www.smarterservices.com/smarterproctoring

https://online.odu.edu/online-experience/coursework/exams-and-proctors/smarterproctoring

https://tophat.com/classroom/online-proctoring-software/

https://exam.net/

https://examsoft.com/

https://proview.io/

https://www.etsu.edu/helpdesk/documents/tegrity_remote_proctoring_setup.pdf

https://safeexambrowser.org/about_overview_en.html

https://proctor360.com/remote-proctoring-essential-to-e-learning-during-the-covid-19-pandemic/ https://github.com/kmamit5296/Online-Proctoring-System

https://110cumlaude.com

https://www.okta.com/sites/default/files/Examity_User_Guide.pdf

https://isit.arts.ubc.ca/examity/

https://examity.com/

https://blog.mettl.com/top-5-proctoring-solution-providers/

https://mettl.com/en/

https://blog.mettl.com/online-test-proctored-exam/

https://www.aiproctor.com/how-it-works

https://smowl.net/en/

https://home.pearsonvue.com/Test-Owner/Deliver/Online-proctored.aspx

https://warrington.ufl.edu/covid-19/information-for/for-students/honorlock-student-guide/

https://honorlock.com

https://honorlock.kb.help/faculty-policies-and-procedures/

https://umd.service-now.com/itsupport/?id=kb_article_view\&sysparm_article=KB0015453\&sys_

kb_id=48df79bb1b378c50ef518738cd4bcb15

https://umd.service-now.com/itsupport/?id=kb_article_view\&sysparm_article=KB0015450\&sys_

kb_id=4fff5d881b95145036c3a9fbbc4bcb91

https://www.oxagile.com/

https://comprobo.co.uk

All web sites listed in webliography were last accessed on July 22nd, 2020. 
Simone Arnò is a fixed-term scholar in the Dep. of Medicine and Aging Sciences at the University of Chieti-Pescara. His principal areas of interest concerns technologies applied to psychological clinical and research field, and the neuroscience investigation.

Alessandra Galassi is a fixed-term scholar in the Dep. of Life, Health and Environmental Sciences at the University of L'Aquila.

Marco Tommasi is a full researcher at the University of Chieti-Pescara, Italy. He took his Ph.D. in scientific psychology at the University of Padua, Italy. He was a research assistant at the "Justus Liebig" University of Giessen, Germany. His actual area of interests are psychophysics, psychometrics and individual differences, in particular, intelligence, personality, emotions, psychological well-being, psychological testing and data analysis. He is currently teaching "Theory and techniques of psychological testing" and "Methodology of research in psychology and multivariate data analysis" in psychological courses. His scientific productions include 39 papers published on indexed journals, books and handbooks of psychological tests. He also developed expertise in programming and computerized adaptive testing.

Aristide Saggino is Professor of Psychometrics at the Department of Medicine and Aging Sciences of the Università di Chieti - Pescara, Italy. He has an extensive background in the area of assessment, individual differences and behavioural - cognitive psychotherapy. He has authored about 19 books and books chapters in Italian and English. Furthermore, he has authored about 85 scientific papers in Italian and English journals.

Pierpaolo Vittorini is Aggregate Professor in Computer Science (INF/01) in the Dep. of Life, Health and Environmental Sciences of the University of L'Aquila. His research interests are computational epidemiology, statistical processing of biomedical data, health information systems and artificial intelligence. He teaches Computer science and Health informatics in the Faculty of Medicine of the University of L'Aquila. He received the "Giuseppe Carruba" award as the best professor of the Faculty of Medicine for the Academic Year 2006/2007. He was the Project Coordinator of the TERENCE EU research project (grant nr. 257410), as well as scientific responsible for other projects funded by companies working in the biomedical sector. He is author of more than one hundred publications on international and national journals, conferences and workshops. He has been a member of the scientific committee of several international conferences and workshops (e.g., ebTEL, mis4TEL, PACBB) and editor of special issues of international journals (e.g., "Evidence- and User-based Technology Enhanced Learning" of IJTEL). 